\title{
Quantitative evaluation of retinal microvasculature and retrobulbar vessels after intravenous chemotherapy for retinoblastoma
}

\author{
Xiuqian $\mathrm{Yi}^{1,2+}$, Jie Sun ${ }^{1,2 \dagger}$, Jiang Qian ${ }^{1,2}$, Jie Guo ${ }^{1,2}$ and Kang Xue ${ }^{1,2^{*}}$
}

\begin{abstract}
Backgroud: To evaluate the changes in retinal microvasculature and retrobulbar blood flow, using optical coherence tomography angiography (OCTA) and Color Doppler imaging (CDI) after intravenous chemotherapy (IVC) in patients with retinoblastoma $(\mathrm{RB})$.

Methods: This was a retrospective comparative case control series involving 30 patients. Ten bilateral RB patients that had a preserved eye with extramacular tumours (group I), 10 unilateral RB treated with IVC that had a normal fellow study eye (group II), and 10 age-matched healthy controls. The macular retinal thickness, foveal avascular zone (FAZ) area, and the macular and peripapillary retinal vessel densities (RVD) were measured. The peak systolic and end diastolic velocities of the ophthalmic, central retinal and posterior ciliary arteries were determined. A comparison among the three groups was conducted.

Results: Between the three cohorts, OCTA revealed no significant difference in FAZ area, superficial foveal and parafoveal RVD, deep parafoveal RVD and peripapillary RVD, $(P>0.05)$. By contrast, the mean deep foveal RVD, the full, inner and outer foveal and the parafoveal retinal thickness were significantly lower in group I compared with the controls, $(P=0.0329,0.0153,0.03110 .0352,0.0215)$. No significant difference in the blood flow velocities occurred in the retrobulbar circulation $(P>0.05)$.

Conclusions: In patients with retinoblastoma, OCTA did not detect significant changes of retinal thickness and vessel density in the eyes treated with IVC, but a slight reduction in retinal thickness and the deep foveal RVD seemed to occur in bilateral RB eyes. The retrobulbar blood flow parameters showed no measurable changes.
\end{abstract}

Keywords: Retinoblastoma, Retrobulbar ocular blood flow, Optical coherence tomography angiography, Intravenous chemotherapy

*Correspondence: xuekang@foxmail.com

${ }^{\dagger}$ Xiuqian Yi and Jie Sun contributed equally to this work.

${ }^{2}$ Key Laboratory of Myopia of the State Health Ministry, and Key

Laboratory of Visual Impairment and Restoration of Shanghai, China, Shanghai, China

Full list of author information is available at the end of the article

\section{Backgroud}

Retinoblastoma (RB) is the most common, primary, intraocular malignancy in children. The improvement in globe salvage is mostly related to the introduction of intravenous chemotherapy (IVC, chemoreduction) coupled with focal consolidation as the most commonly used strategy for RB worldwide [1, 2]. In recent years, advances in treatment options, such as highly selective original author(s) and the source, provide a link to the Creative Commons licence, and indicate if changes were made. The images or other third party material in this article are included in the article's Creative Commons licence, unless indicated otherwise in a credit line to the material. If material is not included in the article's Creative Commons licence and your intended use is not permitted by statutory regulation or exceeds the permitted use, you will need to obtain permission directly from the copyright holder. To view a copy of this licence, visit http://creativecommons.org/licenses/by/4.0/. The Creative Commons Public Domain Dedication waiver (http://creativeco mmons.org/publicdomain/zero/1.0/) applies to the data made available in this article, unless otherwise stated in a credit line to the data. 
intra-arterial have reduced the use of systemic chemotherapy [3]. Although there can be systemic toxicities associated with IVC, such as bone marrow suppression and hearing loss $[4,5]$, limited studies have reported on ophthalmic changes after IVC using spectral domain optical coherence tomography (OCT) [6] and optical coherence tomography angiography (OCTA) [7].

OCTA is a novel, non-invasive method and its recent use, along with the split-spectrum amplitude-decorrelation angiography (SSADA) algorithm, permits the visualisation of the vascular system of the retina at the capillary level. It has also been used to study the microvasculature effects of benign and malignant intraocular tumours [8-10].

In the previous study, we demonstrated that intra-arterial chemotherapy (IAC) had a measurable effect on the retrobulbar blood flow [11]. However, there are limited reports in the literature about CDI changes of these vessels after IVC. In this study, we evaluated the effects of IVC on the retrobulbar and ocular blood flow parameters in patients with retinoblastoma via CDI and OCTA, to understand the underlying ocular toxicities in the paediatric population.

\section{Methods}

\section{Ethics approval and consent to participate}

The Institutional Review Board at Eye, Ear, Nose, and Throat Hospital of Fudan University approved this study. Written informed consent was obtained from the parents, caretakers, or guardians on behalf of all of the children.

\section{Patients}

The retrospective study was conducted at the Department of Ophthalmology, Eye, Ear, Nose and Throat Hospital of Fudan University in Shanghai, China, from January to December 2020. Consecutive patients with RB treated with IVC and enucleation performed earlier in life and currently between the ages of 7 and 15 years were enrolled in this study. The authors divided the eyes into three groups: bilateral RB patients that had a preserved eye with extramacular tumours, with the staging as Group A-C at diagnosis (group I, $n=10$ ), unilateral RB treated with IVC that had a normal fellow study eye (group II, $n=10$ ), and age-matched healthy controls (control group, $n=10$ ).

Subjects with any of the following were excluded: newly diagnosed RB cases and those with less than 1 year follow-up time following the complete stoppage of systemic chemotherapy and/or local therapy; patients who had received external beam radiotherapy (brachytherapy) and subconjunctival chemotherapy, intraarterial or intravitreal chemotherapy; with tumours affecting the macular and optic disc region (edge of tumour was less than $3 \mathrm{~mm}$ from fovea and optic disc boundary); or with macular subretinal fluid, cystoid macular edema, vitreous haemorrhage or cataract.

\section{Ophthalmological examination}

All the children had a full history taken which included their age at the time of the present study, sex, age at the time of first presentation of RB, duration of follow-up, family history, modality of treatment used, number of IVC cycles, international classification of retinoblastoma (ICRB), number of tumours, distance to optic disc (mm) and to foveal $(\mathrm{mm})$. All patients underwent comprehensive ophthalmologic examinations, which included best-corrected visual acuity (BCVA), refraction measurement using auto refraction, spherical equivalence (SE) calculation, slit-lamp biomicroscopy, dilated fundus examination and axial length (AL) measured with an optical biometry device (IOLMaster; Carl Zeiss AG, Jena, Germany).

\section{Optical coherence tomography angiography}

OCTA was employed using a spectral domain system RTVue-XR Avanti (Optovue, Fremont, CA, USA). This system has an A-scan rate of $70 \mathrm{kHz}$ per second with a light source centred at a wavelength of $840 \mathrm{~nm}$ and a bandwidth of $45 \mathrm{~nm}$ [12-14]. Two volumetric raster scans, including one horizontal priority $(x$-fast) and one vertical priority ( $\mathrm{y}$-fast), were obtained consecutively for each area and repeated again. The SSADA algorithm was employed to improve the signal-to-noise ratio by splitting the spectrum to generate multiple repeat OCT frames from the two original repeat OCT frames [15], and any motion artefacts were removed with 3-D orthogonal registration and the merging of the two scans.

Macular $(6 \times 6 \mathrm{~mm})$ and optic disc $(4.5 \times 4.5 \mathrm{~mm})$ OCT angiography scans were acquired. The vessel densities in the foveal, parafoveal and peripapillary areas were assessed as follows. The foveal area was a $1 \mathrm{~mm}$ diameter circle centred on the fovea. The parafoveal area was defined as an annulus with an outer diameter of $3 \mathrm{~mm}$ and an inner diameter of $1 \mathrm{~mm}$ centred on the fovea. The peripapillary area was defined as a $700 \mu \mathrm{m}$ wide elliptical annulus extending outward from the optic disc boundary. The retinal vessel densities of the specific areas were defined as the percentage area occupied by vessels in the corresponding segmented areas [13].

At the same time, the FAZ area and macular thicknesses were measured using the same OCTA system. The FAZ was automatically outlined and measured by built-in ImageJ software. The macular thicknesses, including the foveal and parafoveal area, were obtained using the retinal map protocol. The full retinal thickness was measured 
from the internal limiting membrane to the middle of the retinal pigment epithelium and the inner retinal thickness from the internal limiting membrane to the outer boundary of the inner plexiform layer. The retinal thicknesses of each area were automatically determined using the system's software and were defined as the mean thicknesses of each area.

\section{Colour Doppler imaging measurements}

The HDI 5000 CDI (Philips Ultrasound, Bothell, Washington, USA) with a $7.5 \mathrm{MHz}$ linear probe was used to evaluate the blood flow of retrobulbar vessels posterior ciliary artery (PCA), central retinal artery (CRA) and ophthalmic artery $(\mathrm{OA}))$. The patients were placed in the supine position and examined by the same experienced sonographer. All processes were done in accordance with the CDI measurements protocol, as described previously [16]. The peak systolic velocity (PSV) and end diastolic velocity (EDV) in each vessel were measured. The pulsatility (PI) and resistance (RI) indices were calculated automatically by the scanner.

\section{Statistical analyses}

For the analyses of the data, the Statistical Package for the Social Sciences version 17.0 (SPSS, Chicago, IL) was used, and the descriptive statistics included the standard deviations of the means for all of the variables. The categorical variables were compared using unpaired $S$ tudent 's t-test, $\mathrm{X}$ or Fischer's tests, and the continuous variables between the groups were compared using the MannWhitney U and Kruskal-Wallis tests. $P<0.05$ was considered statistically significant.

\section{Results}

\section{Basic ophthalmic assessment}

Overall, 30 patients (30 eyes) of three groups were included, with a mean age of $9.4 \pm 2.2$ years (range, 7-15years). The demographic and clinical characteristics of the RB patients and healthy controls are summarised in Table 1. There was no significant difference between the three groups concerning their age, visual acuity and axial length $(P>0.05)$. In addition, the duration from last IVC to time of the exam and the number of IVC cycles were similar between group I and II $(P>0.05)$. The age at the time of the first presentation of RB was significantly younger in group I $(P<0.05)$.

\section{OCTA parameters, retinal thickness and retinal vessel densities (RVD)}

Comparing group I with the control group, the retinal vessel densities in the deep foveal was significantly lower in group I (34.9\% vs $39.3 \%, P=0.0329)$. The full, inner and outer foveal and parafoveal retinal thickness
Table 1 The demographic and clinical data of the patients with $\mathrm{RB}$ and healthy controls

\begin{tabular}{lllll}
\hline & Group I & Group II & Control & P value \\
\hline Number of subjects & 10 & 10 & 10 & \\
Age (years) & $9.7 \pm 2.4$ & $8.8 \pm 2.3$ & $9.6 \pm 1.7$ & \\
Gender, $\mathrm{n}$ & & & & \\
$\quad$ Male & 5 & 4 & 6 & \\
$\quad$ Female & 5 & 6 & 4 & \\
Tumor consolidation, $\mathrm{n}$ & & & & \\
$\quad$ Cryotherapy & 8 & 0 & 0 & \\
$\quad$ TTT & 9 & 0 & 0 & \\
$\quad$ Cryotherapy $+\mathrm{TTT}$ & 7 & 0 & 0 & $\mathbf{0 . 0 0 0 3}$ \\
BCVA (logMAR) & 0 & 0 & 0 & \\
Axial Length (mm) & $23.0 \pm 1.1$ & $22.9 \pm 0.8$ & $23.1 \pm 0.7$ & \\
First presentation time & $12.9 \pm 7.7$ & $30.1 \pm 9.6$ & & \\
(mouth) (mouth) & & & & \\
Duration time (year) & $8.2 \pm 2.4$ & $7.3 \pm 2.4$ & & \\
IVC times & $6.5 \pm 1.6$ & $6.5 \pm 1.6$ & & \\
Family history, n (\%) & $1(10 \%)$ & 0 & & \\
\hline
\end{tabular}

Values are expressed as the mean \pm SD. $p<0.05$ was considered statistically significant

$B C V A$ Best-corrected visual acuity, logMAR Logarithm of the minimum angle of resolution, IVC Intravenous chemotherapy. Duration time Duration from last IVC to time of the exam

were significantly thinning in group I (230.0 vs 250.7 , $P=0.0153 ; 50.7$ vs $56.4, P=0.0311 ; 189.3$ vs 206.0 , $P=0.0352 ; 300.7$ vs $325.7, P=0.0215)$. However, there was no significant difference in the FAZ area, the RVD in the superficial foveal and parafoveal area, the RVD in the deep parafoveal area and the RVD in the peripapillary area (all $P>0.05$ ) (Table 2) (Figs. 1 and 2).

Comparing group I with II, and group II with the control group respectively, there were no statistically significant differences in the FAZ area, the RVD in the superficial foveal and parafoveal area, the RVD in the deep foveal and parafoveal area and the RVD in the peripapillary area. The full, inner and outer foveal and parafoveal retinal thickness were similar $(P>0.05$; Table 2$)$.

\section{Retrobulbar blood flow parameters}

The mean retrobulbar blood flow values of the OA, PCA, and CRA among the three groups are shown in Table 3. There were no statistically significant differences in all the parameters.

\section{Discussion}

Choroidal thinning and ischemic atrophy after the treatment of intraarterial chemotherapy in RB have been documented in vivo using EDI-OCT. [17] Our previous results suggested that some significant decreases in the blood flow velocities occurred in the retrobulbar 
Table 2 Retinal Thicknesses and Vessel Densities of three groups

\begin{tabular}{|c|c|c|c|c|c|c|}
\hline \multirow[t]{2}{*}{ Group } & \multirow[t]{2}{*}{1} & \multirow[t]{2}{*}{ II } & \multirow[t]{2}{*}{ Controls } & \multicolumn{3}{|c|}{$P$ value } \\
\hline & & & & | vs II & I vs C & II vs C \\
\hline \multicolumn{7}{|l|}{ Fovea thickness ( $\mu \mathrm{m})$} \\
\hline Full & $230.0 \pm 19.5$ & $244.5 \pm 15.5$ & $250.7 \pm 14.7$ & 0.082 & 0.0153 & 0.3709 \\
\hline Inner & $50.7 \pm 4.3$ & $53.8 \pm 7.4$ & $56.4 \pm 6.4$ & 0.2670 & 0.0311 & 0.4117 \\
\hline Outer & $189.3 \pm 21.2$ & $201.8 \pm 10.0$ & $206.0 \pm 9.4$ & 0.1090 & 0.0352 & 0.3460 \\
\hline Parafoveal thickness ( $\mu \mathrm{m})$ & $300.7 \pm 29.9$ & $321.8 \pm 17.6$ & $325.7 \pm 9.6$ & 0.0704 & 0.0215 & 0.5461 \\
\hline Choroidal thickness & $266.4 \pm 84.7$ & $288.3 \pm 84.1$ & $312.6 \pm 82.7$ & 0.5690 & 0.2330 & 0.5230 \\
\hline FAZ area $(\mathrm{mm} 2)$ & $0.257 \pm 0.088$ & $0.247 \pm 0.133$ & $0.237 \pm 0.058$ & 0.8450 & 0.5559 & 0.8299 \\
\hline \multicolumn{7}{|l|}{$\mathrm{SCP}(\mathrm{RVD}, \%)$} \\
\hline foveal & $21.4 \pm 4.4$ & $21.3 \pm 7.8$ & $23.1 \pm 3.8$ & 0.9722 & 0.3674 & 0.5201 \\
\hline Parafoveal & $45.9 \pm 5.4$ & $49.5 \pm 3.8$ & $49.5 \pm 3.3$ & 0.1018 & 0.0888 & 1.0000 \\
\hline \multicolumn{7}{|l|}{$\mathrm{DCP}(\mathrm{RVD}, \%)$} \\
\hline foveal & $34.9 \pm 4.5$ & $37.1 \pm 9.4$ & $39.3 \pm 4.0$ & 0.5129 & 0.0329 & 0.5045 \\
\hline Parafoveal & $49.8 \pm 5.8$ & $51.5 \pm 3.1$ & $51.1 \pm 2.8$ & 0.4244 & 0.5313 & 0.7655 \\
\hline Peripapillary (RVD, \%) & $50.7 \pm 3.5$ & $53.3 \pm 1.9$ & $52.5 \pm 2.2$ & 0.0537 & 0.1854 & 0.2910 \\
\hline pRNFL & $119 \pm 32.1$ & $124 \pm 12.0$ & $117 \pm 12.4$ & 0.6501 & 0.8562 & 0.2158 \\
\hline
\end{tabular}

Values are expressed as the mean \pm SD. $P<0.05$ was considered statistically significant

$R V D$ Retinal vessel density, SCP Capillary plexus, DCP Deep capillary plexus

circulation in some arteries after IAC [11]. Although IVC has been widely used in RB treatment for over 20 years, limited previous observations of ocular toxicities, including retinal ischemia after IVC, have been published. Shields et al [7] reported a slight reduction in capillary density of the deep capillary plexus in patients after IVC for RB without alterations in the central macular thickness or choroidal thickness. However, there are few reports in the literature about CDI changes of these vessels after IVC.

In this study, we documented that the deep foveal RVD were significantly lower in the preserved eye with extramacular tumours, while the full, inner and outer foveal and the parafoveal retinal thickness were also significantly decreased. Standard IVC for RB includes vincristine and etoposide, and carboplatin, and the related toxicities are minimal. CRA and PCA, branches of OA, supply blood to the inner retina and choroid layer, respectively, through which the chemotherapeutic drugs enter the eyeballs. In our study, there were no measurable changes in the blood flow velocities in the PCA, CRA or OA. These drugs have not yet been observed to cause direct ocular toxicities in RB patients. Perhaps chemotherapy-induced micro-ischemia is below the threshold of causing blood flow velocity changes and visual acuity changes. Instead, IAC may trigger vascular toxicity through endothelial cell inflammation and leukostasis, leading to ocular and orbital vessel toxicity $[18,19]$. IVC continues to have a safe ocular toxicity profile and work as a major treatment for early stage retinoblastoma. The current indications for IVC include patients with bilateral $\mathrm{RB}$, confirmed germline mutation, familial retinoblastoma, aged 4 months or younger and high-risk histopathologic features $[20,21]$.

In this study, eyes without tumours that received chemotherapy (because of the opposite eye with the tumour [group II]) showed no signs of microischemia in OCTA or blood flow velocity changes in CDFI compared with the normal control. While the bilateral RB patients that had a preserved eye with extramacular tumours showed significantly decreased deep foveal RVD and alterations in the full, inner and outer foveal and the parafoveal retinal thickness. We interpreted this finding to represent an effect of focal consolidation or a tumour effect rather than a chemotherapy effect. The focal consolidation, including cryotherapy and transpupillary thermotherapy (TTT), might result in retinal and choroidal thinning and atrophy. Even the focal consolidation was conducted in the extramacular region, it might still have a measurable effect on the deep foveal RVD, and the mild subclinical retinal ischemia was detected only by OCTA.

Azza et al. [6] proposed that retinoblastoma eyes, characterised by thinning of the central fovea, GCL, GCC compared with the control group, and the changes may be due to the genetic defects in RB. Our results proposed that the full, inner and outer foveal and parafoveal retinal thickness were significantly thinning in group I. Bilateral RB patients that had a preserved eye with extramacular tumours, which had genetic defects, and were consistent with the results of Azza. We also 

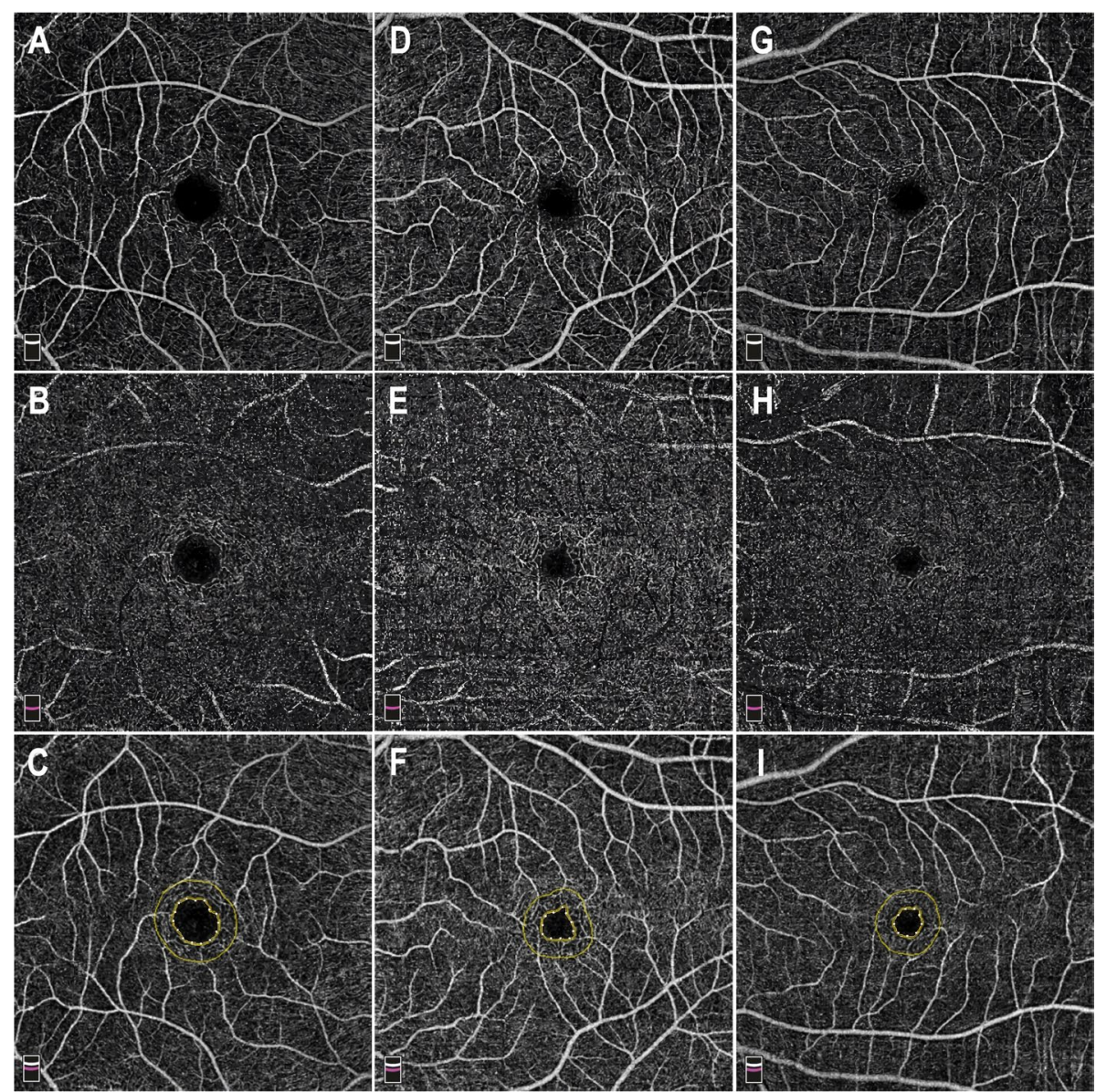

Fig. 1 The example of optical coherence tomography angiography (OCTA) image in three groups. En face image $(6 \times 6 \mathrm{~mm})$ of the corresponding layer of the superficial (A) and deep (B) capillary plexuses, and the area of the foveal avascular zone (FAZ) (C) was automatically calculated in Group I (0.445) (right eye, bilateral RB had a preserved eye with extramacular tumors). En face of the corresponding layer of the superficial (D) and deep (E) capillary plexuses, and the area of the foveal avascular zone (FAZ) (F) in Group II (0.224) (left eye, unilateral RB with a normal eye). En face of the corresponding layer of the superficial $(\mathbf{G})$ and deep (H) capillary plexuses, and the area of the foveal avascular zone (FAZ) (I) in control Group (0.203) (left eye). Capillary density was calculated as percent of vessels per en face image for both plexuses. The mean superficial plexus capillary density did not demonstrate statistically significant difference in three groups. By contrast, mean deep plexus capillary density was significantly reduced in group I compared with controls

compared the vessel densities in the peripapillary area, resulting in no significant difference among the three groups, while RNFL thicknesses and peripapillary RVD were also similar in all three groups which has not been reported before. The vessels in the peripapillary area originate from two systems, the central retinal artery and the short posterior ciliary arteries. The chemotherapy might not have had a measurable vascular toxicity, since vessel densities and the RNFL remained unchanged when chemotherapeutic drugs entered the eyeballs.

This study was not without certain limitations. First of all, we must mention that the number of participants included was limited to reach a statistically significant difference. Second, since the difference remained slight between the RB tumour and the control groups, a larger study may be required to validate this outcome. Additionally, OCTA with analyses is an examination that we could not perform in the supine position as it needs a patient's cooperation. So far, therefore, it has not been possible to perform OCTA on patients under anaesthesia and on young infants, and the retinal thickness and vessel density before IVC treatment could not be detected. Finally, as IAC is more popular in the treatment of advanced $\mathrm{Rb}$ which seem to have more ocular toxicities, further study will be investigated on retinal microvasculature after IAC. 

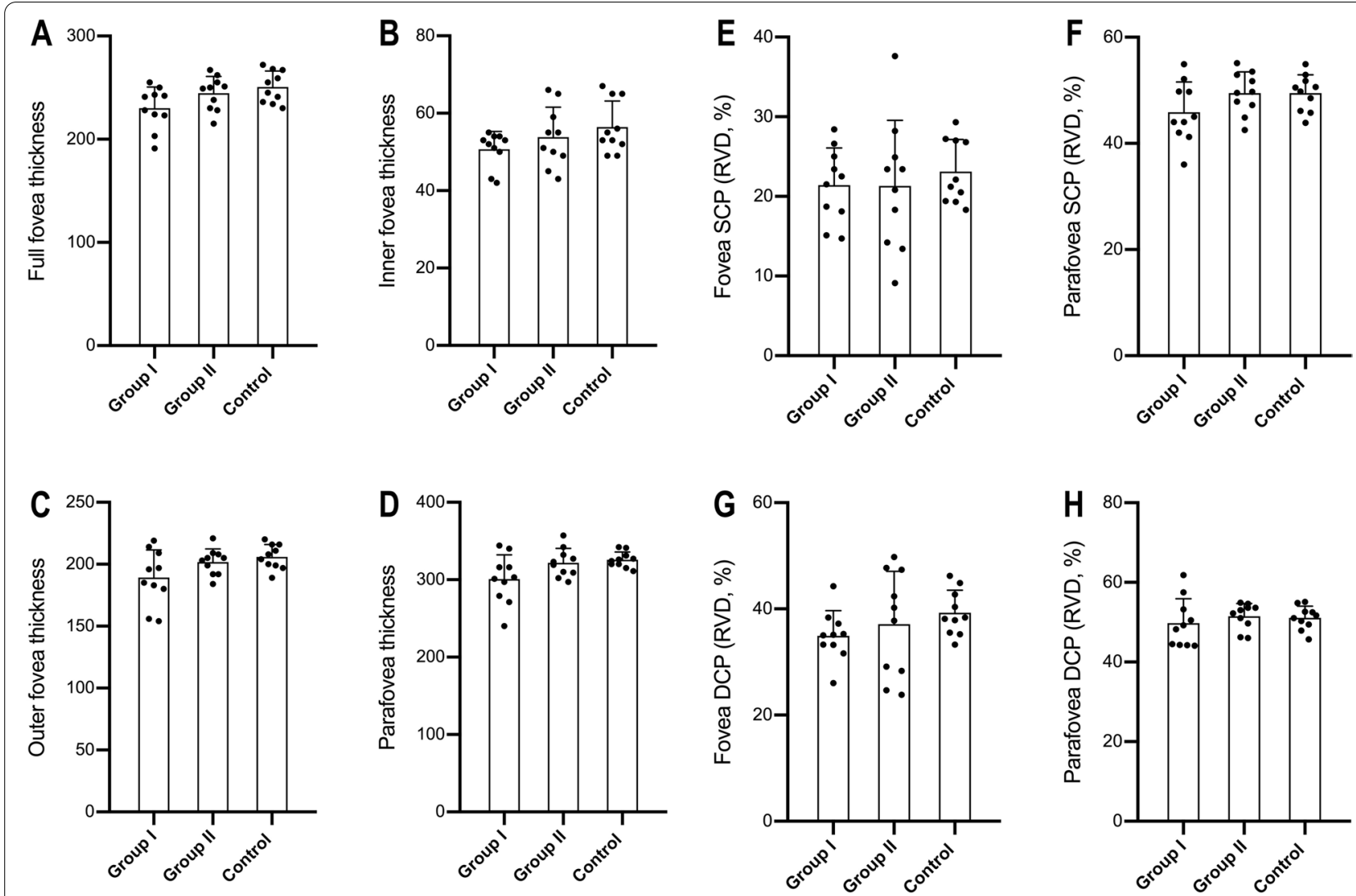

Fig. 2 A comparison among the three groups was conducted, including the macular retinal thickness (a full foveal, b inner foveal, c outer foveal, d parafoveal), foveal avascular zone (FAZ) area, and the macular and peripapillary retinal vessel densities. As the retinal vessel densities (RVD) in superficial foveal (e) and parafoveal (f), the deep foveal $(\mathbf{g})$ and deep parafoveal (h). By contrast, the mean deep foveal RVD, the full, inner and outer foveal and the parafoveal retinal thickness were significantly lower in group I compared with the controls, $(P=0.0329,0.0153,0.03110 .0352,0.0215)$

Table 3 Hemodynamic Doppler parameters in the OA, PCA, and CRA of Eyes After Intravenous Chemotherapy for RB

\begin{tabular}{llllll}
\hline Parameter & Group I & Group II & Controls & P value & \\
\cline { 5 - 6 } & & & & I vs II & I vs C \\
\hline Central retinal artery & & & & 0.7644 \\
PSV (cm/s) & $11.28 \pm 3.52$ & $11.52 \pm 3.73$ & $11.72 \pm 2.92$ & 0.8840 & 0.8953 \\
EDV (cm/s) & $3.82 \pm 1.42$ & $3.52 \pm 1.64$ & $3.71 \pm 1.84$ & 0.6671 & 0.8827 \\
RI & $0.66 \pm 0.07$ & $0.69 \pm 0.08$ & $0.68 \pm 0.09$ & 0.3839 & 0.5859 \\
PI & $1.12 \pm 0.23$ & $1.18 \pm 0.21$ & $1.15 \pm 0.21$ & 0.5500 & 0.7642 \\
POsterior ciliary artery & & & & 0.7958 \\
PSV (cm/s) & $11.94 \pm 3.08$ & $12.21 \pm 3.24$ & $12.52 \pm 3.43$ & 0.8507 & 0.6954 \\
EDV (cm/s) & $3.67 \pm 1.18$ & $3.84 \pm 1.64$ & $3.73 \pm 1.48$ & 0.7932 & 0.9213 \\
RI & $0.69 \pm 0.09$ & $0.69 \pm 0.10$ & $0.70 \pm 0.10$ & 1.0000 & 0.8168 \\
PI & $1.06 \pm 0.23$ & $1.04 \pm 0.25$ & $1.04 \pm 0.22$ & 0.8544 & 0.8447 \\
Ophthalmic artery & & & & 0.8377 \\
PSV (cm/s) & $29.40 \pm 6.63$ & $30.47 \pm 5.63$ & $31.47 \pm 5.6$ & 0.7018 & 0.8256 \\
EDV (cm/s) & $6.82 \pm 2.62$ & $7.11 \pm 2.34$ & $6.98 \pm 3.12$ & 0.7970 & 0.4604 \\
RI & $0.77 \pm 0.08$ & $0.76 \pm 0.09$ & $0.78 \pm 0.10$ & 0.7958 & 0.9025 \\
PI & $1.25 \pm 0.38$ & $1.24 \pm 0.40$ & $1.27 \pm 0.43$ & 0.9549 & 0.8078 \\
\hline
\end{tabular}




\section{Conclusion}

In summary, our results suggested that in retinoblastoma patients, OCTA did not detect significant changes of the retinal thickness or vessel density in eyes treated with IVC, but a slight reduction in the foveal and parafoveal retinal thickness, and deep foveal RVD seemed to occur in bilateral RB eyes. These changes might be due to the effect of focal consolidation or the genetic defects in RB. No measurable changes in the blood flow velocities occurred in the retrobulbar circulation after IVC with a long-term follow-up.

\begin{abstract}
Abbreviations
OCTA: Optical coherence tomography angiography; CDI: Color Doppler imaging; IVC: Intravenous chemotherapy; RB: Retinoblastoma; FAZ: Foveal avascular zone; RVD: Retinal vessel densities; OCT: Optical coherence tomography; SSADA: Split-spectrum amplitude-decorrelation angiography; IAC: Intra-arterial chemotherapy; BCVA: Best-corrected visual acuity; SE: Spherical equivalence; AL: Axial length; PCA: Posterior ciliary artery; CRA: Central retinal artery; OA: Ophthalmic artery; PSV: Peak systolic velocity; EDV: End diastolic velocity; PI: Pulsatility indices; RI: Resistance indices.
\end{abstract}

\section{Acknowledgements}

All authors met the ICMJE authorship criteria. Neither honoraria nor payments were made for authorship.

\section{Authors' contributions}

Dr. Xue had full access to all of the data in the study and take responsibility for the integrity of the data and the accuracy of the data analysis. All authors read and approved the final manuscript. Study concept and design: Xue, Yi, Sun, Guo, Qian. Acquisition, analysis, or interpretation of data: Xue, Yi, Sun Drafting of the manuscript: Yi, Xue. Critical revision of the manuscript for important intellectual content: All authors. Statistical analysis: Yi, Sun. Obtained funding: Xue, Qian. Administrative, technical, or material support: Xue, Qian.

\section{Funding}

Project supported by the Science and Technology Commission of Shanghai Municipality, China (Grant No.20Y11911200). The funder had no role in the design and conduct of the study; collection, management, analysis, and interpretation of the data; preparation, review, or approval of the manuscript; and decision to submit the manuscript for publication.

\section{Availability of data and materials}

All of our detailed information were collected from doctor's workstation of Eye and ENT Hospital of Fudan University, which is read-only, so we are sorry that the detailed data can't be shared.

\section{Declarations}

\section{Ethics approval and consent to participate}

The Institutional Review Board at Eye, Ear, Nose, and Throat Hospital of Fudan University approved this study.

\section{Consent for publication}

Not applicable. Images in our article are entirely unidentifiable and there are no details on individuals reported within the manuscript.

\section{Competing interests}

The authors have declared that no competing interests exist.

\section{Author details}

${ }^{1}$ Department of Ophthalmology, Eye, Ear, Nose, and Throat Hospital of Fudan University, Shanghai 200031, China. ${ }^{2}$ Key Laboratory of Myopia of the State Health Ministry, and Key Laboratory of Visual Impairment and Restoration of Shanghai, China, Shanghai, China.
Received: 31 August 2021 Accepted: 10 November 2021

Published online: 26 November 2021

\section{References}

1. Shields CL, De Potter P, Himelstein BP, Shields JA, Meadows AT, Maris JM. Chemoreduction in the initial management of intraocular retinoblastoma. Arch Ophthalmol. 1960;114(11):1330-8. Pubmed: 8906023. https:// doi.org/10.1001/archopht.1996.01100140530002.

2. MacCarthy A, Birch JM, Draper GJ, Hungerford JL, Kingston JE, Kroll ME, et al. Retinoblastoma: treatment and survival in Great Britain 1963 to 2002. Br J Ophthalmol. 2009;93(1):38-9. Pubmed: 1883841. https://doi. org/10.1136/bjo.2008.139626.

3. Abramson DH, Shields CL, Munier FL, Chantada GL. Treatment of retinoblastoma in 2015: agreement and disagreement. JAMA Ophthalmol. 2015;133(11):1341-7. Pubmed: 26378747. https://doi.org/10.1001/jamao phthalmol.2015.3108.

4. Lambert MP, Shields C, Meadows AT. A retrospective review of hearing in children with retinoblastoma treated with carboplatin-based chemotherapy. Pediatr Blood Cancer. 2008;50(2):223-6. Pubmed: 17278120. https:// doi.org/10.1002/pbc.21155.

5. Qaddoumi I, Bass JK, Wu J, Billups CA, Wozniak AW, Merchant TE, et al. Carboplatin-associated ototoxicity in children with retinoblastoma. J Clin Oncol. 2012;30(10):1034-41. https://doi.org/10.1200/JCO.2011.36.9744.

6. Said AMA, Elbayomi AM, Shaat AAK. Structural changes of the macula and optic nerve head in the remaining eyes after enucleation for retinoblastoma: an optical coherence tomography study. BMC Ophthalmol. 2017;17(1):251. https://doi.org/10.1186/s12886-017-0650-9.

7. Sioufi K, Say EAT, Ferenczy SC, Leahey AM, Shields CL. Optical coherence tomography angiography findings of deep capillary plexus microischemia after intravenous chemotherapy for retinoblastoma. Retina (Philadelphia, Pa). 2019;39(2):371-8. https://doi.org/10.1097/IAE.00000 00000001973.

8. Valverde-Megías A, Say EA, Ferenczy SR, Shields CL. Differential macular features on optical coherence tomography angiography in eyes with choroidal nevus and melanoma. Retina (Philadelphia, Pa). 2017;37(4):73140. https://doi.org/10.1097/IAE.0000000000001233.

9. Li Y, Say EA, Ferenczy S, Agni M, Shields CL. Altered parafoveal microvasculature in treatment-naive choroidal melanoma eyes detected by optical coherence tomography angiography. Retina (Philadelphia, Pa). 2017;37(1):32-40. https://doi.org/10.1097//AE.0000000000001242.

10. Sioufi K, Say EAT, Ferenczy SC, Shields CL. Parafoveal microvascular features on optical coherence tomography angiography in eyes with circumscribed choroidal hemangioma. Retina (Philadelphia, Pa). 2018;38(6):1091-9. https://doi.org/10.1097//AE.0000000000001709.

11. Xue K, Liu A, Hui R, Zhang J, Qian J. Retrobulbar ocular blood flow changes measured by colour Doppler imaging after intra-arterial chemotherapy in retinoblastoma. Br J Ophthalmol. 2018;101(10):1419-22. https://doi.org/10.1136/bjophthalmol-2016-310056.

12. Jia Y, Tan O, Tokayer J, Potsaid B, Wang Y, Liu JJ, et al. Split-spectrum amplitude-decorrelation angiography with optical coherence tomography. Opt Express. 2012;20(4):4710-25. https://doi.org/10.1364/OE.20.004710.

13. Yu J, Jiang C, Wang X, Zhu L, Gu R, Xu H, et al. Macular perfusion in healthy Chinese: an optical coherence tomography angiogram study. Invest Ophthalmol Vis Sci. 2015;56(5):3212-7. https://doi.org/10.1167/ iovs.14-16270.

14. Jia Y, Wei E, Wang X, Zhang X, Morrison JC, Parikh M, et al. Optical coherence tomography angiography of optic disc perfusion in glaucoma. Ophthalmology. 2014;121(7):1322-32. https://doi.org/10.1016/j.ophtha. 2014.01.021.

15. Jia Y, Morrison JC, Tokayer J, Tan O, Lombardi L, Baumann B, et al. Quantitative OCT angiography of optic nerve head blood flow. Biomed Optics Express. 2012;3(12):3127-37. https://doi.org/10.1364/BOE.3.003127.

16. Founti P, Harris A, Papadopoulou D, Emmanouilidis P, Siesky B, Kilintzis $\checkmark$, et al. Agreement among three examiners of colour Doppler imaging retrobulbar blood flow velocity measurements. Acta Ophthalmol. 2011;89(8):e631-4. https://doi.org/10.1111/j.1755-3768.2011.02236.x.

17. Maidana DE, Pellegrini M, Shields JA, Shields CL. Choroidal thickness after intraarterial chemotherapy for retinoblastoma. Retina (Philadelphia, Pa). 2014;34(10):2103-9. https://doi.org/10.1097/IAE.0000000000000190. 
18. Steinle JJ, Zhang Q, Thompson KE, Toutounchian J, Yates CR, Soderland $C$, et al. Intra-ophthalmic artery chemotherapy triggers vascular toxicity through endothelial cell inflammation and leukostasis. Invest Ophthalmol Vis Sci. 2012;53(4):2439-45. https://doi.org/10.1097/IAE.0000000000 000190.

19. Tse BC, Kaste SC, Brennan R, Orr B, Wilson MW. Enophthalmos and choroidal atrophy after intraophthalmic artery chemotherapy for retinoblastoma. Ophthalmology. 2015;122(2):435-7. https://doi.org/10.1016/j. ophtha.2014.09.021.

20. Shields CL, Lally SE, Leahey AM, Jabbour PM, Caywood EH, Schwendeman R, et al. Targeted retinoblastoma management: when to use intravenous, intra-arterial, periocular, and intravitreal chemotherapy. Curr Opin Ophthalmol. 2014;25(5):374-85. https://doi.org/10.1097//CU.0000000000 000091.

21. Chévez-Barrios P, Eagle RC Jr, Krailo M, Piao J, Albert DM, Gao Y, et al. Study of unilateral retinoblastoma with and without Histopathologic high-risk features and the role of adjuvant chemotherapy: a Children's oncology group study. J Clin Oncol. 2019;37(31):2883-91. https://doi.org/ 10.1200/JCO.18.01808.

\section{Publisher's Note}

Springer Nature remains neutral with regard to jurisdictional claims in published maps and institutional affiliations.

- fast, convenient online submission

- thorough peer review by experienced researchers in your field

- rapid publication on acceptance

- support for research data, including large and complex data types

- gold Open Access which fosters wider collaboration and increased citations

- maximum visibility for your research: over 100M website views per year

At BMC, research is always in progress.

Learn more biomedcentral.com/submissions 